\title{
INVERSIONES ECONÓMICAS GAMIFICADAS COMO SOPORTE PARA LA RETROALIMENTACIÓN ENTRE PARES
}

Gamified economic investments for supporting feedback in peer-assessment Investimentos económicos gamificados como suporte ao feedback entre pares

\author{
Juan Fraile (1) \\ Patricia Ruiz Bravo (2) \\ María Valdivieso (3) \\ (1) Universidad Francisco de Vitoria, España. Teléfono: +34 650052657. Correo electrónico: \\ juan.fraile@ufv.es \\ (2) Universidad Francisco de Vitoria, España. Teléfono: +34 639583753. Correo electrónico: \\ p.ruiz.prof@ufv.es \\ (3) Universidad Autónoma de Madrid, España. Teléfono: +34 690797046. Correo electrónico: \\ maria4.valdivieso@gmail.com
}

\section{Resumen}

Esta experiencia de evaluación formativa se basa en la retroalimentación entre iguales entre grupos de estudiantes en relación al desarrollo de su trabajo final de la asignatura. Esta práctica se llevó a cabo en una asignatura del tercer curso en educación universitaria. Inicialmente, los estudiantes se explican entre sí lo que están llevando a cabo, habiendo una primera retroalimentación entre ellos. Posteriormente, el grueso de la actividad se basa en que cada grupo invierte al menos un billete de 100, 200 y 500 euros en los proyectos del resto de equipos, en función de que lo que les haya gustado. Asimismo, por la parte de atrás de cada billete se proporciona retroalimentación explicando qué puntos fuertes tiene el proyecto y también qué partes debería mejorar y cómo.

Palabras clave: Evaluación entre iguales; retroalimentación; aprendizaje cooperativo

\begin{abstract}
This formative assessment experience is based on peer-feedback between groups of students regarding their final project of the course. Teachers performed this practice in a course of the third year in higher education. Firstly, students explain each other the work they are performing and give mutually the first feedback in this activity. Later on,
\end{abstract}


the bulk of the activity, each group invested at least one 100, 200 and 500 euros note in the projects of the rest of the teams according to their preferences and opinions. Moreover, on the back of the notes, students give feedback regarding strengths, weaknesses, and possible solutions for that project.

Keywords: Peer-assessment; feedback; cooperative learning

\section{Resumo}

Esta experiência de avaliação formativa é baseada na retroalimentação entre grupos de alunos em relação ao desenvolvimento do seu trabalho final da disciplina. Esta prática foi realizada numa disciplina do terceiro ano do ensino universitário. Inicialmente, os alunos explicam uns aos outros o que estão a fazer, havendo uma primeira retroalimentação entre eles. Posteriormente, a maior parte da atividade é baseada no investimento de cada grupo nos projetos das outras equipas, atribuindo uma nota de 100, 200 e 500 euros, dependendo do que eles gostaram. Além disso, no verso de cada nota é dada retroalimentação, explicando quais os pontos fortes do projeto e também, quais as partes que devem ser melhoradas e como.

Palavras-chave: Avaliação de pares; feedback; aprendizagem cooperativa

\section{Introducción}

Esta experiencia de evaluación formativa en educación superior se basa en la evaluación entre pares o iguales trabajando los estudiantes entre sí. Esta práctica no sólo tiene un objetivo de recepción de retroalimentación por parte de los estudiantes cuyo trabajo es juzgado (Sadler, 2010), sino que también supone un aprendizaje para los estudiantes que proporcionan esa retroalimentación al tener que identificar fortalezas y debilidades y facilitar soluciones a sus compañeros (Bouwer, Lesterhuis, Bonne, \& De Maeyer, 2018). Asimismo, otro pilar de la experiencia es la gamificación -empleo de los elementos del juego en un contexto no lúdico- con el fin de incrementar la motivación hacia esta nueva experiencia educativa (Hanus \& Fox, 2015).

\section{Contextualización}

Esta actividad se ha desarrollado en una asignatura de 6 créditos ECTS del tercer curso del Grado en Ciencias de la Actividad Física y del Deporte. Se llevó a cabo en dos 
grupos de 50 estudiantes aproximadamente y dos profesores de la asignatura -a la vez-; y en otro grupo con 15 estudiantes y un profesor. Esta dinámica se encuadra dentro del desarrollo del trabajo final de la asignatura (50\% de la calificación final) en el que los estudiantes tienen que diseñar y llevar a cabo una gamificación aplicada a cualquier contexto y edad relacionados con nuestros estudios. El proyecto se fue desarrollando a lo largo de varias sesiones aplicando los contenidos de la asignatura. Tras algunas sesiones en las que cada grupo de estudiantes había diseñado una buena base para su gamificación, se llevó a cabo esta dinámica.

\section{Diseño y desarrollo}

El objetivo fue desarrollar el pensamiento crítico y creativo para poder aportar un valor a los demás compañeros $\mathrm{y}$, por tanto, obtener nuevas ideas y retroalimentación para su propio proyecto. El medio a través del que proporcionar la retroalimentación al resto de equipos fue a través de billetes de 100, 200 y 500 euros (Figura 1). Por un lado, son un billete normal, por el otro, hay dos preguntas: “¿Qué puntos fuertes tiene?” y “¿Cuáles son sus puntos débiles y cómo podría mejorarlos?”.
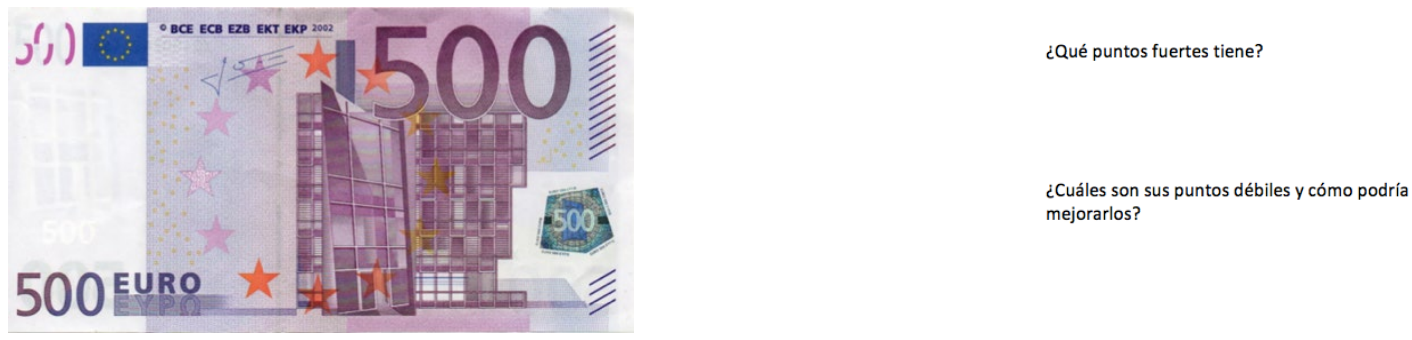

Figura 1. Billete de 500 euros para proporcionar retroalimentación.

\section{Desarrollo}

La experiencia comenzó con la dinámica cooperativa 'círculo dentro-círculo fuera' con la que se instó a que los estudiantes comunicasen su proyecto a otros compañeros de diferentes grupos de trabajo. La disposición inicial de los estudiantes es de dos círculos concéntricos, teniendo cada uno una pareja enfrente, en el otro círculo. Cada estudiante debía exponer su proyecto. La duración de esta exposición fue de un minuto seguido de un minuto y medio en el que el receptor de la información pudo aportar ideas, preguntarle más y entablar un diálogo. Después se cambian los roles con el mismo funcionamiento. Tras terminar, uno de los círculos gira en un sentido para establecer 
nuevas parejas. Este proceso se llevó a cabo tres veces. Tras estas rondas, cada equipo ya conocía el resto de los proyectos -al menos uno de sus componentes-. A partir de este momento cada equipo tenía dos funciones que se repartieron -y podían cambiar- durante 15 minutos. Quedarse al lado de una infografía sobre su proyecto para seguirlo explicando o ir a otros grupos para seguir informándose y proporcionar su retroalimentación informalmente. Tras estos minutos, durante los cinco minutos siguientes, tenían que escribir su retroalimentación en los billetes. Tenían un número de billetes de distinto valor igual al número de equipos (menos uno, el suyo). Se colocó un celo verticalmente al lado de cada infografía de los equipos. Así, los grupos fueron pegando los billetes. Evidentemente, el valor del billete también era una retroalimentación que indicaba su mayor o menor predilección hacia los diferentes proyectos llevando a cabo una ronda de inversores con un proyecto ganador.

\section{Evaluación}

La evaluación de la experiencia se realizó, primariamente, a partir de los comentarios de los estudiantes al terminar la sesión. Este proceso se trasladó a los estudiantes no sólo como una forma de recoger su opinión por interés del docente, sino como una forma de hacerles partícipes en un proceso crítico como elemento de aprendizaje. Por ello, se les instó a que propusieran alternativas y no sólo hicieran comentarios superficiales sobre aquellos que les habían parecido adecuados o no. A partir de sus opiniones, también se reunieron los docentes para registrar una valoración final de la actividad.

En relación al planteamiento, no todos entregan todos los billetes, derivado principalmente del problema con el tiempo que, además, afecta tanto a la calidad de la retroalimentación como a la importancia que se otorga a este proceso. Asimismo, el factor competitivo conlleva no ser éticos en ocasiones, aunque no hubiera ninguna recompensa. Se propone meter los billetes en un sobre o caja, pero por el contrario uno de los aspectos motivantes es la visualización de los billetes al lado de cada equipo. Igualmente, los estudiantes declaran que este aspecto visual propicia una cierta tendencia a partir de los billetes que ya se ven dados a un equipo.

En la parte final de la actividad, se genera cierto alboroto y mezcla de funciones. Al terminar, igualmente, se podría haber hecho una revisión más importante de la retroalimentación recibida. Asimismo, algunos estudiantes consideran que no fue muy 
útil debido a que no pudieron comprender o explicar el proyecto en profundidad o las razones por las que se habían tomado ciertas decisiones.

\section{Conclusiones}

El diseño de esta actividad, parcialmente gamificada, de retroalimentación entre pares responde al objetivo de aprendizaje y mejora de la tarea objetivo junto al trabajo de otras competencias. Todos los estudiantes participan simultáneamente y cara a cara, aspecto importante de la evaluación entre pares (Panadero \& Alqassab, 2019). Las mejoras identificadas permitirán un mejor aprovechamiento de la actividad ya que el principal problema, como apunta Patton (2012), es que los estudiantes suelen percibir que la retroalimentación recibida por sus compañeros es poco útil y superficial.

\section{Referencias}

Bouwer, R., Lesterhuis, M., Bonne, P., \& De Maeyer, S. (2018). Applying criteria to examples or learning by comparison: Effects on students' evaluative judgment and performance in writing. Frontiers in Education, 3, 1-12.

Hanus, M. D., \& Fox, J. (2015). Assessing the effects of gamification in the classroom: A longitudinal study on intrinsic motivation, social comparison, satisfaction, effort, and academic performance. Computers \& Education, 80, 152-161.

Panadero, E., \& Alqassab, M. (2019). An empirical review of anonymity effects in peer assessment, peer feedback, peer review, peer evaluation and peer grading. Assessment \& Evaluation in Higher Education, 1-26. Recuperado de https://www.tandfonline.com/doi/abs/10.1080/02602938.2019.1600186

Patton, C. (2012). 'Some kind of weird, evil experiment': student perceptions of peer assessment. Assessment \& Evaluation in Higher Education, 37(6), 719-731.

Sadler, D. R. (2010). Beyond feedback: Developing student capability in complex appraisal. Assessment \& Evaluation in Higher Education, 35(5), 535-550. 